\title{
Eighteen-month outcome of pulmonary valve stent implantation by direct right ventricle puncture: An animal study
}

\author{
Gang-Jun Zong, MD, PhD, ${ }^{\mathrm{a}}$ Yuan Bai, MD, ${ }^{\mathrm{b}}$ Ming-Biao Gu, MD, ${ }^{\mathrm{b}}$ Hai-Bin Jiang, MD, ${ }^{\mathrm{b}}$ Hong Wu, MD, \\ $\mathrm{PhD},{ }^{\mathrm{b}}$ Xian-Xian Zhao, $\mathrm{MD}, \mathrm{PhD},{ }^{\mathrm{b}}$ and Yong-Wen Qin, $\mathrm{MD}, \mathrm{PhD}^{\mathrm{b}}$
}

Objective: The purpose of this study was to investigate the feasibility and safety of pulmonary valve implantation via direct right ventricle puncture.

\begin{abstract}
Methods: A standard thoracotomy and direct right ventricle puncture were performed in 8 healthy sheep to implant the pulmonary valve stents. Animals were followed up for 18 months.

Results: Three sheep died within the first 4 months after stent placement. The remaining 5 animals survived. After 18 months, examinations by color echocardiography, 64-slice computed tomography scan, and cardiac catheter showed an ideal position of each stent. The function of the pulmonary valves and hearts was not different compared with the preoperative conditions of the sheep. Anatomic examination revealed that the stent was covered by a layer of endothelial tissue with no stent fracture or valvular calcification. The histologic evaluation of the stent and surrounding tissue showed that the surface of the stent was smooth and covered by a complete layer of endothelial cells without obvious infiltration of inflammatory cells. The vascular wall was integrative without tear phenomenon in each layer of tissue.
\end{abstract}

Conclusions: These results show that pulmonary valve stents can be implanted via direct right ventricle puncture. Further studies evaluating xenograft valve material and the effect of implantation in vivo are needed. (J Thorac Cardiovasc Surg 2011;141:518-22)

Since Bonhoeffer and colleagues ${ }^{1}$ successfully performed percutaneous pulmonary valve replacement in a 12-year-old boy with right ventricular outflow tract (RVOT) stenosis and pulmonary valve insufficiency in October 2000, a flurry of clinical and experimental percutaneous valve replacement studies ensued. ${ }^{2-4}$ Today, these studies continue to be popular. ${ }^{5,6}$ Because the diameter of the sheath for implanting valve stents is relatively large, it is easy to damage the peripheral veins, particularly in pediatric patients. ${ }^{7}$ In addition, this approach can also damage the heart chambers, resulting in cardiac tamponade. ${ }^{8}$ The sheath needs to be manipulated to a certain degree to ensure the stent is ultimately placed in the correct position. Therefore, to avoid extracorporeal circulation in cardiac surgery and to prevent damage to the peripheral blood vessels or heart chambers when using a large sheath during percutane-

From the Department of Cardiology, ${ }^{\text {a }} 101$ Hospital of PLA, Wuxi, Jiangsu Province, China; and the Department of Cardiology, ${ }^{\mathrm{b}}$ Changhai Hospital, the Second Military Medical University, Shanghai, China.

This research was supported by the National High Technology Research and Development Program of China ("863"Program) (2006AA02Z4D7).

Conflicts of Interest: None.

Disclosures: Authors have nothing to disclose with regard to commercial support.

G.-J. Zong and Y. Bai contributed equally to this study.

Received for publication June 11, 2009; revisions received July 30, 2009; accepted for publication Aug 26, 2009.

Address for reprints: Yong-Wen Qin, MD, PhD, Department of Cardiology, Changhai Hospital, Second Military Medical University, Shanghai, 200433, China (E-mail: ywqinsmmu@yahoo.cn).

0022-5223/\$36.00

Copyright (c) 2011 by The American Association for Thoracic Surgery doi:10.1016/j.jtcvs.2009.08.065 ous valve replacement, the efficacy of pulmonary valve implantation via direct right ventricle puncture was investigated.

\section{MATERIALS AND METHODS \\ Pulmonary Valve Stent}

The pulmonary valve stent was constructed with an artificial valve and a conduit-shaped stent as previously reported ${ }^{9,10}$ and shown in Figure 1, $A$ and $B$.

\begin{abstract}
Animals
Eight healthy sheep ( 5 male and 3 female), weighing an average of $23.5 \pm$ $3.1 \mathrm{~kg}$, were used. No abnormalities were found by preoperative electrocardiography, thoracic radiography, and color echocardiography examinations. Animals were deprived of water and food for 10 hours preoperatively. Animals were anaesthetized initially by intramuscular injection of ketamine (10 $\mathrm{mg} / \mathrm{kg}$ body weight), followed by an intravenous injection of vecuronium bromide ( $2 \mathrm{mg})$. All animals were intubated and ventilated during the procedure. Propofol $(0.2 \mathrm{mg} / \mathrm{kg} / \mathrm{min})$ was administered intravenously by a continuous rate infusion to maintain the anesthetic condition. All animals received care in compliance with the Guide for the Care and Use of Laboratory Animals, published by The National Academies in 1988.
\end{abstract}

\section{Valve Stent Implantation and Postoperative Treatment}

A 10-cm incision was made in the subxiphoid, and a lower partial sternotomy was performed. The pericardium was suspended, and the right ventricular free wall was exposed. An echocardiography transducer was placed directly on the main pulmonary artery to measure the diameter of the pulmonary artery throughout the procedure. The size of the stent was selected on the basis of the diameter ratio of the pulmonary artery to the stent lumen. The desired ratio was 1:1.1. A purse-string suture was placed in the anterior wall of the right ventricle near its apex. A sheath was punctured directly into 


\section{Abbreviations and Acronyms \\ $\mathrm{H} \& \mathrm{E}=$ hematoxylin-eosin \\ RVOT $=$ right ventricular outflow tract}

the right ventricle cavity within the purse string in the direction of the outflow tract. The delivery route for the stent was established by introducing a J-type steel guidewire via the sheath (Figure 2, B). The entire procedure was performed under transthoracic echocardiography guidance. The position of the guidewire could be noted when it made contact with the pulmonary artery wall. The guidewire was subsequently advanced into the distal segment of the left or right pulmonary artery. The sheath was withdrawn, and a $10 \mathrm{~F}$ expandable sheath was deployed into the right ventricle via the guidewire to expand the puncture hole. After the expandable sheath was removed, a 14F delivery sheath with a nickel-titanium valve stent was inserted into the pulmonary artery via the guidewire. After confirming that the stent had been placed in the optimal position under echo guidance, the delivery sheath and guidewire were removed. The purse-string suture was tied. Echocardiography was performed to evaluate the prosthetic valve function and hemodynamic function. The thoracic cavity was then closed.

After the procedure, all animals were given an intramuscular injection with penicillin $(800,000$ units) and subcutaneous injections of low molecular heparin ( $2500 \mathrm{IU}$ ) for 3 days. Oral aspirin ( $3 \mathrm{mg} / \mathrm{kg}$ body weight) was given for 30 days.

\section{Anatomic Study Immediately After Valve Stent Implantation}

Right ventricular angiography was performed in the animals with successful valve stent implantation to assess the stent position. One sheep with ideal valve stent implantation was sacrificed by femoral artery bleeding 12 hours postoperatively. The right ventricle was opened to observe the stent position and structure of the surrounding tissues.

\section{Angiography and Anatomic Examination 18 Months After Stent Implantation}

The remaining sheep underwent right ventricle angiography to evaluate the stent position 18 months after implantation under digital subtraction angiography (Siemens, Munich, Germany). In addition, 64-slice enhanced computed tomography scans were performed in 2 animals.

Surviving sheep were sacrificed at 18 months by femoral artery bleeding. Pulmonary arterial tissues were collected for histologic examination. Some tissue samples containing nitinol wire were plastic-embedded, sliced with a gold stainless-steel microtome blade, and stained with hematoxylin-eosin (H\&E). Samples that did not contain nitinol wire were paraffin-embedded, sectioned, and stained with H\&E.

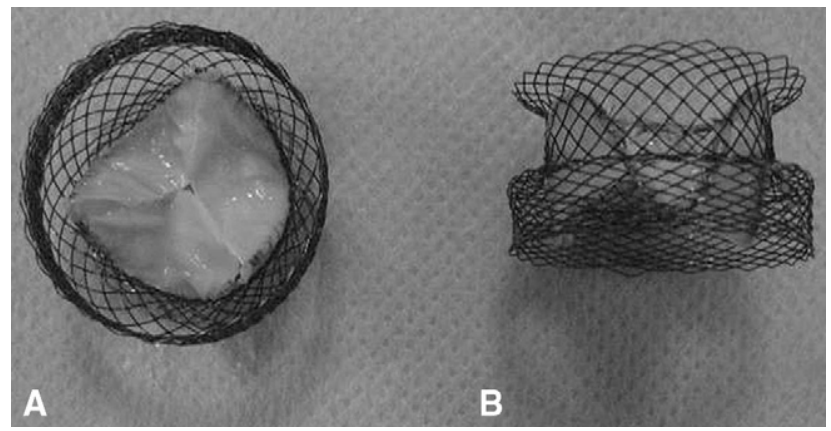

FIGURE 1. Pulmonary valve stent construction, A, Anterior view of pulmonary valve stent. B, Lateral view of pulmonary valve stent.

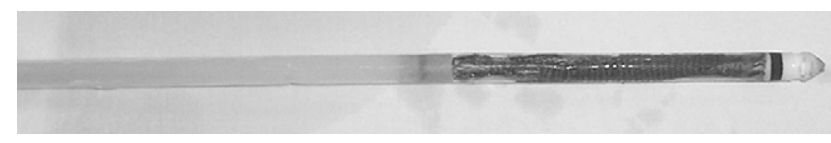

FIGURE 2. Valved stent completely crimped into a $14 \mathrm{~F}$ sheath.

\section{RESULTS}

\section{Operative Success}

Eight sheep underwent stent placement. In 1 sheep, the stent was too small and dropped into the right ventricle. The animal died of severe bleeding when the stent was withdrawn from the right ventricle. Stent implantation was successful in the remaining 7 sheep; however, 1 sheep died 3 hours postoperatively, presumably of anesthesia-related complications. Another sheep had shortness of breath and exercise intolerance postoperatively. Despite 7 days of diuretic treatment, this sheep died (4 months postoperatively), and thrombosis in the proximal region of the stent was identified on postmortem examination. All remaining 5 animals survived. One animal was sacrificed 12 hours postoperatively. The remaining 4 sheep recovered and resumed a normal diet and preoperative level within 3 to 5 days after the operation.

Intraoperative echocardiography performed after stent placement identified a pulmonary artery diameter of 14.7 $\pm 0.8 \mathrm{~mm}$ and a stent lumen diameter of $19.1 \pm 1.6 \mathrm{~mm}$. The operation duration was $85.4 \pm 6.6$ minutes (Table 1).

\section{Angiography and Anatomy Examination of the Valve Stent Immediately After Implantation}

The position of the stent was ideal in all sheep. Right ventricular angiography (performed immediately before recovery) showed that the stent in 1 sheep was implanted near the RVOT. The end and neck of the stent had obvious contraction and relaxation with the outflow tract (Figure 3, $A$ and $B$ ).

Twelve hours postoperatively, no hematoma or bleeding was identified in the pocket of the right ventricle in the

TABLE 1. Surgical information on implantations performed in experimental sheep

\begin{tabular}{lccc}
\hline ID & $\begin{array}{c}\text { Pulmonary valve annulus } \\
\text { ultrasound }(\mathbf{m m})\end{array}$ & $\begin{array}{c}\text { Diameter of } \\
\text { stent }(\mathbf{m m})\end{array}$ & $\begin{array}{c}\text { Operation } \\
\text { time }(\mathbf{m i n})\end{array}$ \\
\hline 1 & 14.1 & 18 & 85 \\
2 & 13.8 & 18 & 95 \\
3 & 15.6 & 22 & 75 \\
4 & 15.4 & 20 & 80 \\
5 & 15.2 & 20 & 90 \\
6 & 14.8 & 18 & 85 \\
7 & 13.7 & 18 & 88. \\
$8^{*}$ & - & - & - \\
Mean & 14.7 & 19.1 & 85.4 \\
SD & 0.8 & 1.6 & 6.6 \\
Minimum & 13.6 & 18 & 75 \\
Maximum & 15.6 & 22 & 95 \\
\hline
\end{tabular}

$S D$, Standard deviation. *Implantation of valved stent failed in sheep number 8 . 


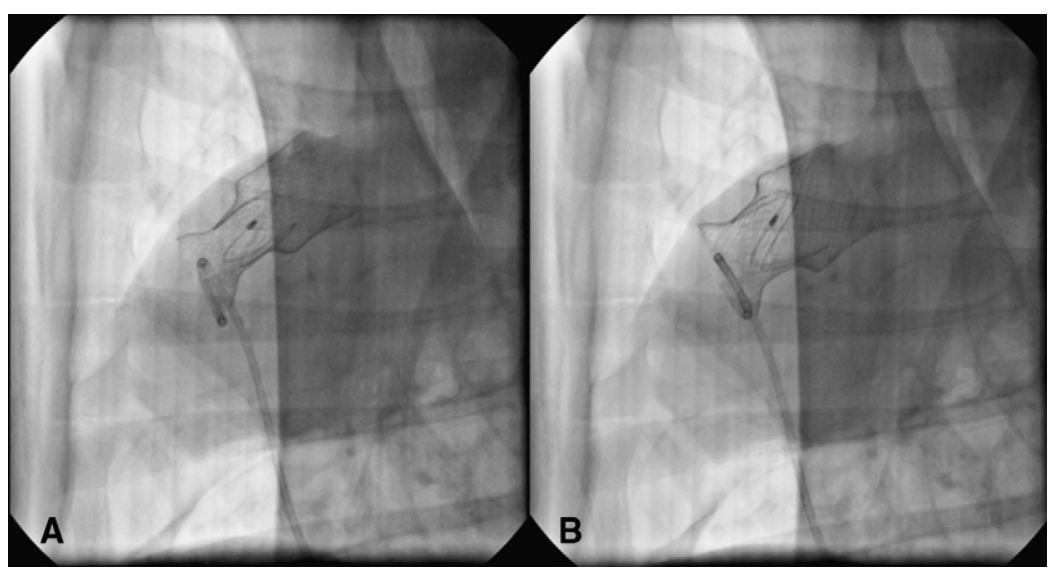

FIGURE 3. Valved stent contracted and relaxed with RVOT contraction. A, One implanted stent contracted with RVOT contraction. The diameter of the stent neck is similar to the diameter of the pigtail catheter tip. B, The same stent relaxed with RVOT relaxation. The diameter of the stent neck is bigger than the diameter of the pigtail catheter tip.

sheep evaluated during the anatomic study (Figure 4, A). The pulmonary valve stent was placed in a good position; the proximal stent flare was fixed at the beginning of the main pulmonary artery and fit perfectly in the RVOT with no impact on tricuspid valve function. The original pulmonary valve was compressed tightly, and no space remained between the stent and the pulmonary arterial wall. There was no thrombosis in the prosthetic valve, and no obvious damage was evident in the RVOT or main pulmonary artery.

\section{Angiography and Anatomy Examination of the Valve Stent 18 Months Postoperatively}

Anatomic study in 4 sheep performed 18 months postoperatively revealed a normal tissue covering the sutures in the right ventricle (Figure 4, B). The stents were in ideal positions and covered by a layer of endothelial tissue (Figure $4, C$ ). The leaves of the prosthetic valve were intact and soft with no evidence of excrescence (eg, thrombosis), calcification, or damage. The sutures on the valve ring and stents themselves were covered by endothelial tissue. No fracture was noted in any stent.
A 64-slice computed tomography scan was performed in 2 of the 4 animals, showing patent stents in good positions (Figure 5).

Anatomy examination performed 18 months postoperatively revealed no valvular calcification. The tissues stained with $H \& E$ showed that the nitinol wires of the stent were smooth and covered by a complete layer of endothelial cells (Figure 6, $A$ and $B$ ). Fibrous tissue hyperplasia was noted in the surrounding area without significant inflammatory reaction. The H\&E-stained pulmonary artery tissue sections that did not contain nitinol wire showed no significant infiltration of inflammatory cells (Figure 6, $C$ and $D$ ). The vascular wall was integrative without tear phenomenon in each layer of tissue.

\section{DISCUSSION}

Our research group previously reported successful percutaneous pulmonary valve replacement by pulmonary valve stent. ${ }^{5}$ After a 6-month follow-up period, the outcome was good. Because the peripheral blood vessels are small in pediatric patients and can be easily damaged during the

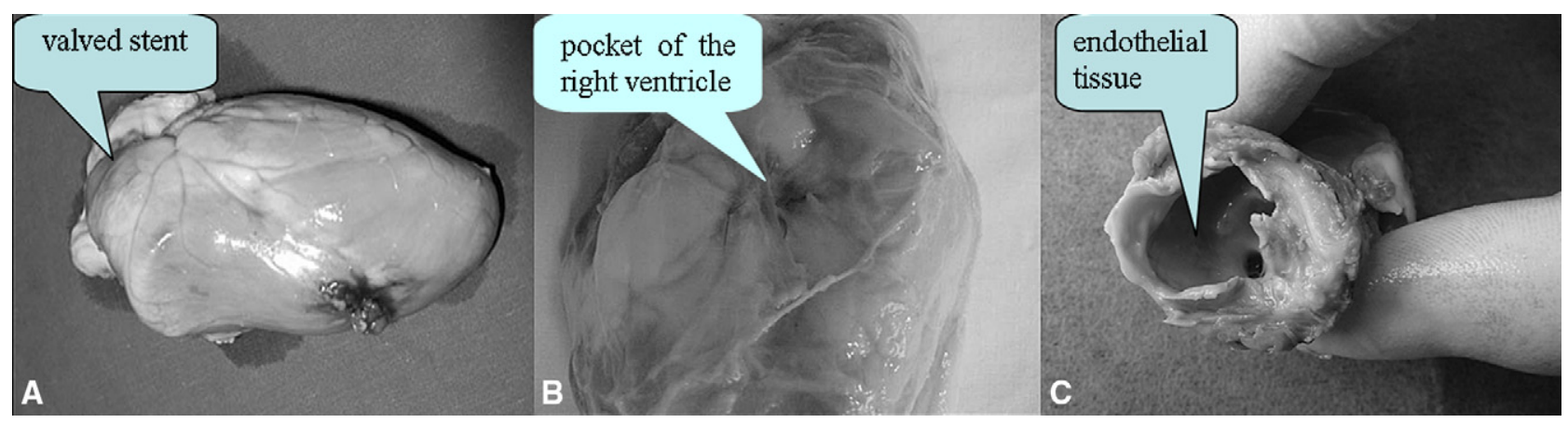

FIGURE 4. Anatomic study performed postoperatively. A, No hematoma or bleeding was identified in the pocket of the right ventricle in the sheep 12 hours postoperatively. B, Anatomic study performed 18 months postoperatively revealed a normal tissue covering over the sutures in the right ventricle. C, Stents were covered by a layer of endothelial tissue. 


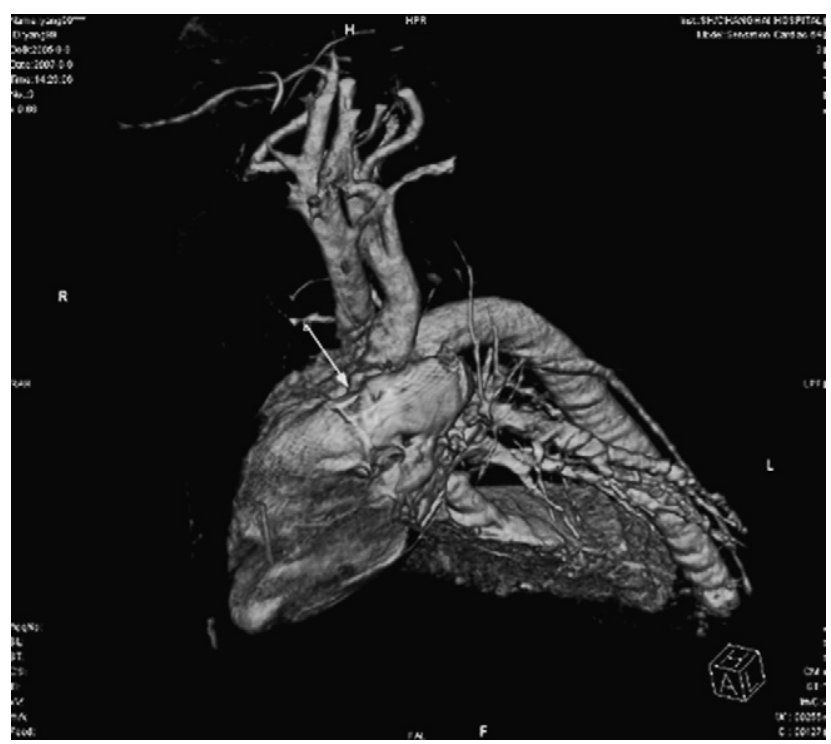

FIGURE 5. Multislice computed tomography scan further confirmed the desired position of the valved stent 18 months after implantation.

insertion of a rough sheath, a minimally invasive surgical method for pulmonary valve replacement via transcatheter thoracotomy could be considered a viable alternative. ${ }^{11}$ In this study, sheep underwent pulmonary valve replacement via transcatheter thoracotomy and were followed up for an 18-month period postoperatively. The results of this pilot study confirm the possibility and reliability of this surgical procedure.

The procedure is performed on the beating heart via right ventricular puncture, exchanging an expandable sheath, and inserting a pulmonary valve stent to finally release the replacement stent. During the operation, color echocardiography is used to measure the diameter of the pulmonary artery and guide the stents to ensure appropriate positioning.

The anatomic study performed 12 hours postoperatively indicated that the stents were ideally placed and fixed properly with no damage to the arterial wall. The study also confirmed that the stent design was reasonable for replacing the original valve with a prosthetic valve. In addition, the operation had no significant impact on cardiac structure. We suggest that this is a feasible technique for clinical practice. The anatomic study performed 18 months postoperatively showed that the ventricular puncture was fully healed and that the stents were in good condition.

One animal had right ventricular failure 4 months after the operation. The animal did not respond to diuretic treatment and finally died of thrombosis in the proximal region of the stent. The possible cause for this development may be related to the low pressure in the right side of the heart, which ultimately increases the risk of thrombosis in the stent or prosthetic valve. In addition, this animal was administered an antiplatelet drug for only 1 month, which may not have been adequate to prevent thrombosis after
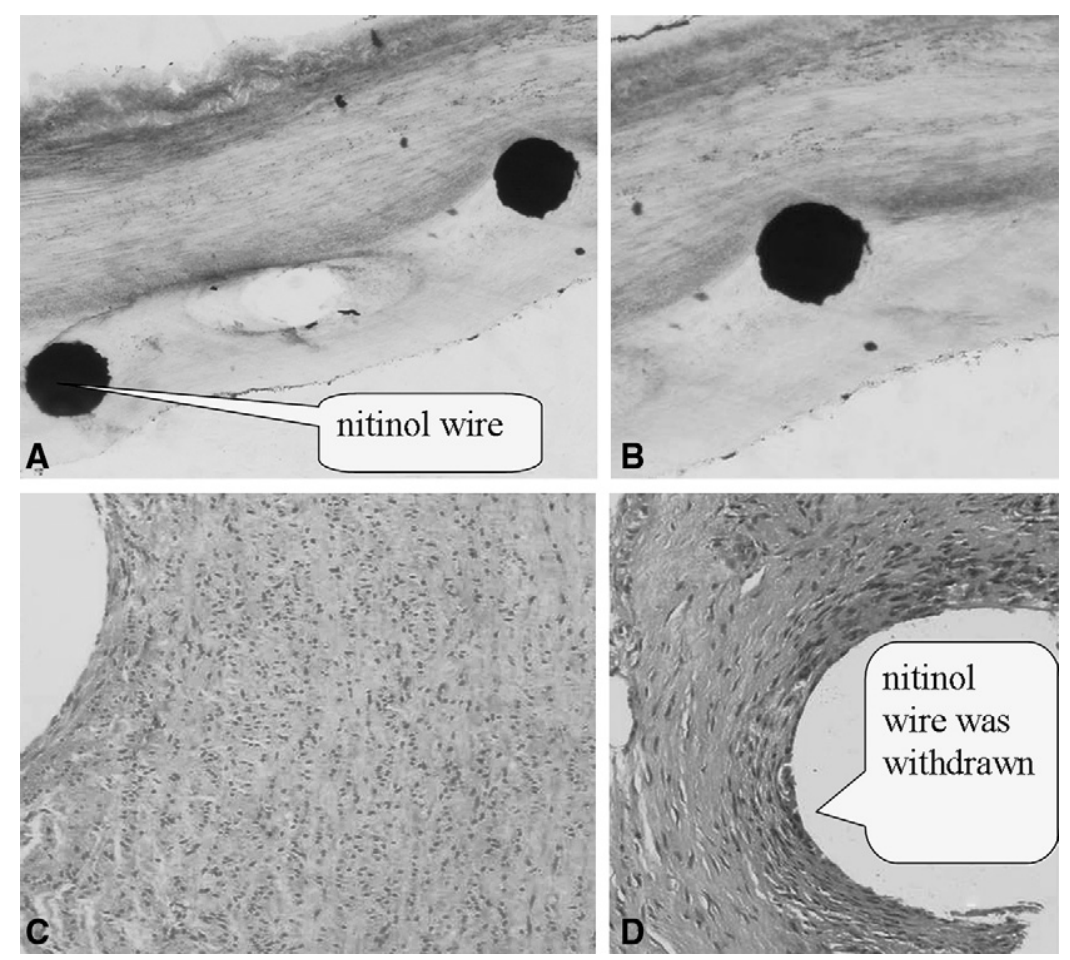

FIGURE 6. A and B, H\&E-stained pulmonary tissues containing nitinol wires and surrounding tissues sliced with a gold stainless-steel microtome blade $(\times 40)$ show a smooth surface of nitinol wire. C, H\&E-stained pulmonary artery wall $(\times 200)$ shows non-tear phenomenon of the vascular wall. D, H\&Estained pulmonary artery tissue without nitinol wire shows no significant infiltration of inflammatory cells. 
valve replacement surgery. We suggest that the standard anticoagulation treatment with warfarin in clinical practice should be considered. At present, there are no reports regarding anticoagulation treatment for prosthetic nickeltitanium valve stent implantation. This topic could benefit from further investigations to improve the long-term safety of valve replacement. ${ }^{12}$

The stent was placed in the RVOT in 1 sheep. Because the outflow tract is a muscular structure, it can contract and relax along with the heart, which may result in metal fatigue after a certain (unspecified) period of time and ultimately lead to the possibility of stent fracture. Theoretically, the original pulmonary valve is the most ideal place for stent positioning; however, in practice, stents are sometimes implanted in an unplanned location regardless of whether color echocardiography or digital subtraction angiography is used to assist in stent positioning. No adverse effects caused by this stent location problem were noted in this sheep for the duration of the study period. The anatomic study performed 18 months postoperatively showed no evidence of stent fracture that might be related to the stent wiring technique used in this study. These results indicate that a small amount of position deviation is not likely to cause any serious complications, perhaps because there are no important anatomic structures in this area that can be negatively affected by a wrongly placed stent, such as the initiation of the coronary artery.

\section{CONCLUSIONS}

Although this is a pilot study, some specific limitations are worth mentioning. The prosthetic valve selected for this experiment was derived from sheep pericardium and is not practicable in the clinical setting. Therefore, it is necessary to perform studies to evaluate xenograft valve material and the effect of implantation in vivo. Second, the valved stent was placed in the native pulmonary artery with a soft wall. Sheep anatomy is dissimilar to that of humans. There was no animal model to mimic a right ventricular-pulmonary artery conduit in this study. Specifically, some patients have large RVOTs after transannular patch repair of tetralogy of Fallot, and the size of the stent should be larger for these patients. Further studies should attempt to create a similar, simple, and reproducible animal model. The small sample size in our study is also an obvious limitation.

\section{References}

1. Bonhoeffer P, Boudjemline Y, Saliba Z, Merckx J, Aggoun Y, Bonnet D, et al. Percutaneous replacement of pulmonary valve in a right-ventricle to pulmonaryartery prosthetic conduit with valve dysfunction. Lancet. 2000;356:1403-5.

2. Nordmeyer J, Coats L, Bonhoeffer P. Current experience with percutaneous pulmonary valve implantation. Semin Thorac Cardiovasc Surg. 2006;18:122-5.

3. Attmann T, Quaden R, Jahnke T, Muller-Hulsbeck S, Boening A, Cremer J, et al. Percutaneous pulmonary valve replacement: 3-month evaluation of selfexpanding valved stents. Ann Thorac Surg. 2006;82:708-13.

4. Khambadkone S, Nordmeyer J, Bonhoeffer P. Percutaneous implantation of the pulmonary and aortic valves: indications and limitations. J Cardiovasc Med. 2007;8:57-61.

5. Attmann T, Quaden R, Freistedt A, König C, Cremer J, Lutter G. Percutaneous heart valve replacement: histology and calcification characteristics of biological valved stents in juvenile sheep. Cardiovasc Pathol. 2007;16:165-70.

6. Lurz P, Coats L, Khambadkone S, Nordmeyer J, Boudjemline Y, Schievano S, et al. Percutaneous pulmonary valve implantation impact of evolving technology and learning curve on clinical outcome. Circulation. 2008;117:1964-72.

7. Kostolny M, Tsang V, Nordmeyer J, Van Doorn C, Frigiola A, Khambadkone S, et al. Rescue surgery following percutaneous pulmonary valve implantation. Eur J Cardiothorac Surg. 2008;33:607-12.

8. Feinstein JA, Kim N, Reddy VM, Perry SB. Percutaneous pulmonary valve placement in a 10-month-old patient using a hand crafted stent-mounted porcine valve. Catheter Cardiovasc Interv. 2006;67:644-9.

9. Zong G, Bai Y, Jiang H, Li W, Wu H, Zhao X, et al. Use of a novel valve stent for transcatheter pulmonary valve replacement: an animal study. $J$ Thorac Cardiovasc Surg. 2009;137:1363-9.

10. Bai Y, Zong G, Jiang H, Li W, Wu H, Zhao X, et al. Percutaneous reimplantation of a pulmonary valved stent in sheep: a potential treatment for bioprosthetic valve degeneration. J Thorac Cardiovasc Surg. 2009;138:733-7. Epub 2009 Jun 26.

11. Feldman T, Martin B. Prospects for percutaneous valve therapies. Circulation. 2007;116:2866-77.

12. Coats L, Bonhoeffer P. New percutaneous treatments for valve disease. Heart. 2007;93:639-44. 